\title{
Sonographic Rim in Acute Renal Cortical Necrosis
}

\begin{abstract}
Ultrasonography is the first line of investigation requested for patients presenting with a clinical features of acute renal failure. Acute renal Cortical Necrosis (ACN) is an uncommon cause of acute renal failure secondary to reduced perfusion to the renal cortex with relative sparing of the medulla. There is limited literature available on early sonographic features of acute cortical necrosis. Ultrasonography finding of hypoechoic renal cortex is a diagnostic feature of acute cortical necrosis. This appearance can be aptly called "Sonographic hypoechoic cortical rim". Bilateral ACN following pancreatitis is an extremely rare entity. Due to rarity of association between pancreatitis and bilateral renal cortical necrosis, authors reported case of 25-year-old male presented with severe abdominal pain, vomiting, reduced urine output, constipation. Ultrasonography revealed normal size kidneys with diffuse circumferential hypoechoic appearance of cortex on both sides. Laboratory investigations demonstrated elevated white blood cell count, raised serum amylase. Diagnosis of ACN secondary to acute pancreatitis was made.
\end{abstract}

Keywords: Acute pancreatitis, Sonographic hypoechoic cortical rim, Ultrasonography

\section{CASE REPORT}

A 25-year-old male patient presented to the emergency department with history of severe abdominal pain, vomiting, reduced urine output and constipation for three days. Patient revealed history of binge drinking two-day-ago. No additional relevant history was revealed by the patient. Patient was conscious and oriented. All vitals were stable with pulse of $82 \mathrm{bpm}$ and blood pressure of $118 / 80 \mathrm{mmHg}$ was recorded. Abdominal examination elicited severe epigastric tenderness. Physical and general examination was normal. Ultrasonography performed subsequently revealed normal size kidneys with diffuse circumferential hypoechoic appearance of cortex on both sides [Table/Fig-1a-c]. Both kidneys were of normal size without calculus or hydroureteronephrosis. No cortical calcification was seen on either side. The pancreas was enlarged with loss of surface lobulations, appeared hypoechoic and there was peripancreatic fat stranding [Table/Fig-2a,b]. No free fluid was noted in the abdomen. No other positive finding was noted in the abdomen. Based on above sonographic features, provisional diagnosis of ACN secondary to acute pancreatitis was suggested.

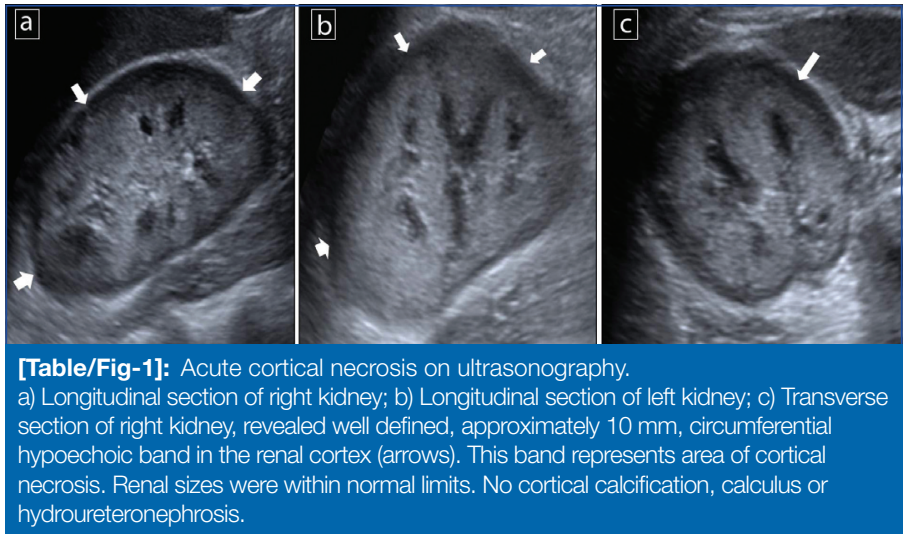

Laboratory investigations demonstrated elevated white blood cell count, raised serum amylase-1326 U/L (normal range: 40-140 U/L), raised serum lipase-900 U/L (normal range: 0-160 U/L) and deranged renal function tests with serum creatinine of $15 \mathrm{mg} / \mathrm{dL}(0.5-1.2 \mathrm{mg} /$ $\mathrm{dL})$. Based on the clinico-radiological correlation, final diagnosis of acute cortical necrosis secondary to acute pancreatitis was confirmed. Patient underwent dialysis twice over next two days. Third day patient
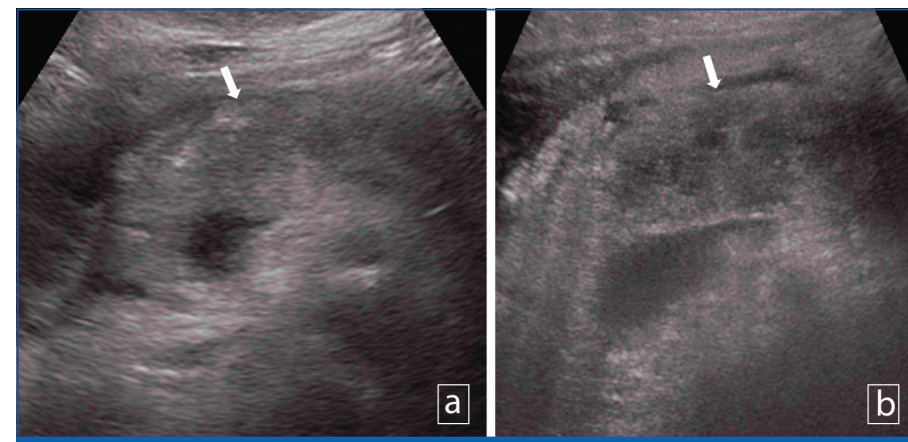

[Table/Fig-2]: Acute pancreatitis on ultrasonography.

a) Transverse ultrasound on curvilinear transducer; b) Transverse ultrasound on linear transducer, shows presence of diffusely bulky pancreas (arrows) with loss of surface lobulations. The pancreas appears heterogeneous in echotexture, predominantly hypoechoic, better appreciated in Fig b. No dilatation of the pancreatic duct noted. No parenchymal calcifications seen. The surrounding fat was echogenic suggestive of inflammation. No acute fluid collections were seen in the peripancreatic region.

developed acute respiratory distress syndrome and died on the fourth day following admission. The postmortem was not performed as no consent was given by patient's relatives.

\section{DISCUSSION}

Each kidney is supplied via respective left or right renal artery arising from the abdominal aorta at L1/L2 vertebral body level. Each main renal artery divides into anterior and posterior divisions. These give rise to five segmental branches near or at hilum namely apical, anteriorsuperior, anterior-inferior, lower and posterior segmental branches. The first four branches arise from the anterior division and posterior segmental artery is a continuation of posterior division of main renal artery. These segmental branches divide into interlobar branches located between medullary pyramids. The interlobar arteries give rise to arcuate branches which curve around the medullary pyramids. These arcuate branches give form vertically oriented intralobular arteries (Cortical radial arteries). The afferent arterioles supplying the glomeruli arise from the interlobular arteries [1,2].

Acute cortical necrosis refers to ischemic necrosis of the renal cortex with relative sparing of the medulla. It accounts for approximately 6-7\% of all cases of acute kidney injury in developing countries; however, the incidence is progressively declining according to recent data [3]. 
It is usually bilateral due to underlying systemic etiologies however, unilateral cases have also been reported [4]. Causes of the acute cortical necrosis can be broadly classified into obstetric (50-70\%) or non-obstetric causes (20-30\%). Obstetric complications include septicabortion, abruptio placentae, severe pre-eclampsia/eclampsia, prolonged intrauterine death and thrombotic microangiopathy of pregnancy. Pregnancy unrelated causes include severe dehydration, burns, sepsis with shock, venom toxin, drugs and toxins, malaria, traumatic blood loss, transfusion reaction, renal transplant rejection and haemolyticuraemic syndrome [3].

Significantly diminished renal arterial perfusion is the final pathway in setting of cortical necrosis. Although the pathophysiology of cortical necrosis is unclear, the proposed inciting factor is intense vasospasm of the small vessels and/or toxin induced endothelial injury. The renal cortex is more prone to injury secondary to greater oxygen tension in this region leading to preferential damage following sudden drop in perfusion. If the vasospasm is brief and flow re-established, it leads to reversible acute tubular necrosis. More prolonged ischemia causes renal cortical necrosis. The cortico-medullary differentiation and subcapsular rim of cortex is spared due to sparing of medullary arterioles and collateral circulation to the subcapsular cortex from capsular vessels [5]. Biopsy is the gold standard for the diagnosis of cortical necrosis, however, due to poor haemodynamic status and coagulopathy biopsy is usually avoided [3]. Currently, diagnosis is based on clinical and laboratory evaluation supported by imaging modalities like CT scan, Magnetic Resonance Imaging (MRI) and angiography.

Contrast Enhanced Computed Tomography (CECT) scan shows specific features: 1) Reverse rim sign- parenchymal phase shows enhancement of the renal medulla and non-enhancing/hypoenhancing cortex; 2) Cortical rim sign- although most of the cortex does not enhance, peripheral 1-2 mm section of cortex shows enhancement due to collateral supply from capsular perforating vessels; 3) Arterial cut-off sign- abrupt termination of the contrast in the renal artery; 4) Delayed phase does not show contrast excretion [6-8]. MRI shows low signal intensity of the inner cortex and the column of Bertin on T1 and T2 weighted sequences [9]. Angiography shows abrupt termination of vascularity at the interlobar and arcuate artery levels which results in irregular scalloped perfusion defects of the renal cortex [10].

Ultrasonography is the first investigation requested for patient with clinical features of renal failure. Sonography findings of ACN mentioned in the literature includes renal enlargement and cortical calcifications. Renal arteries and veins can also be evaluated on sonography without administration of contrast to look for presence of thrombosis. Another important feature of acute cortical necrosis sparsely mentioned in literature is the hypoechoic appearing renal cortex. According to author's literature search, only one study has so farmentioned about hypoechoic appearing cortex on ultrasonography in ACN [11]. This hypoechoic area corresponds to region of cortical necrosis. This finding is seen well before renal enlargement or cortical calcifications, renal sizes being usually within normal limits in the acute phase. Presence and detection of sonographic hypoechoic cortical rim will help in early identification of the patients with acute cortical necrosis for the early and timely initiation of therapy. No other pathology is known to produce hypoechoic cortex on sonography other than acute cortical necrosis, which makes it a diagnostic feature. If patient survives, late findings which can be picked are shrunken kidneys with or without cortical calcifications.

Cortical rim sign mentioned on CECT corresponds to spared and enhancing subcapsular cortex, not the ischemic cortex, hence 'cortical rim sign' on CT and 'sonographic cortical rim' on ultrasound do not corresponds to same areas of cortex. Only few cases of acute cortical necrosis have been so far mentioned in the literature following acute pancreatitis [12-14]. Most of the causes of acute pancreatitis are secondary to hypovolemic, however, in pancreatitis it may be secondary to release of vasoactive or cytotoxic substances [12]. This patient was normotensive initially, however, developed hypovolemic later.

\section{CONCLUSION(S)}

Sonography is frequently the first line radiological investigation performed in patients presenting to emergency department. Diagnosis of acute cortical necrosis can be established early, if the hypoechoic cortical rim is recognised on ultrasonography. This will help in better patient management and avoid further unnecessary radiological work up of the patient like CECT scan. Sonologists attending to emergencies should be aware of this finding as it can be picked up ahead of laboratory parameters of ACN as in present case. Only few cases of acute cortical necrosis have been reported following acute pancreatitis, present case being one of them.

\section{REFERENCES}

[1] Rani N, Singh S, Dhar P, Kumar R. Surgical importance of arterial segments of human kidneys: An angiography and corrosion cast study. Journal of Clinical and Diagnostic Research: JCDR. 2014;8(3):01-03.

[2] Dyer RB, Regan JD, Kavanagh PV, Khatod EG, Chen MY, Zagoria RJ, et al. Percutaneous nephrostomy with extensions of the technique: Step by step. Radiographics. 2002;22(3):503-25.

[3] Prakash J, Singh VP. Changing picture of renal cortical necrosis in acute kidney injury in developing country. World J Nephrol. 2015;4(5):480-86.

[4] Tovbin D, Lantsberg S, Feldman L, Rachinsky I, Lupu L, Hertzanu Y, et al Unilateral acute renal cortical necrosis (ACN) following skipping with a rope. Nephrol Dial Transplant. 2000;15(3):415-18.

[5] Wang M, Wu R, Chen J, Tzeng W, Tsai J. CT findings of acute renal cortical necrosis. Chinese Journal of Radiology-Taipei. 2007;32(2):103-06.

[6] Jordan J, Low R, Jeffrey Jr RB. CT findings in acute renal cortical necrosis. Journal of Computer Assisted Tomography. 1989;14(1):155-56.

[7] Goergen TG, Lindstrom RR, Tan H, Lilley JJ. CT appearance of acute cortical necrosis. AJR Am J Roentgenol. 1981;137:176-77.

[8] Dyer RB, Chen MY, Zagoria RJ. Classic signs in uroradiology. RadioGraphics. 2004;24(Spec Issue):S247-80.

[9] Jeong JY, Kim SH, Lee HJ, Sim JS. Atypical low-signal-intensity renal parenchyma: Causes and patterns. Radiographics. 2002;22(4):833-46.

[10] Tuttle RJ, Minielly JA. The angiographic diagnosis of acute hemorrhagic renal cortical necrosis. Radiology. 1978;126(3):637-38.

[11] Sefczek RJ, Beckman I, Lupetin AR, Dash N. Sonography of acute renal cortical necrosis. American Journal of Roentgenology. 1984;142(3):553-54.

[12] Krishna GSR, Kishore KC, Sriram NP, Sainaresh W, Lakshmi AY, Kumar VS, et al. Bilateral renal cortical necrosis in acute pancreatitis. Indian Journal of Nephrology. 2009;19(3):125.

[13] Anandan AK, Balachandran P, Chowksey A, Sankaranarayanan G, Samuel U. Bilateral renal cortical necrosis following acute pancreatitis- A rare complication of a common disease. Saudi J Kidney Dis Transpl. 2018;29(5):1211-15.

[14] Dave KD, Patel RB, Patel BJ, Solanki SM, Shah BK. A rare entity of acute bilatera cortical necrosis following acute pancreatitis. Med J DY Patil Univ. 2015;8:540-42.

PARTICULARS OF CONTRIBUTORS:

1. Lecturer, Department of Radiodiagnosis, Goa Medical College Bambolim, Goa, India.

2. Assistant Professor, Department of Radiodiagnosis, Goa Medical College Bambolim, Goa, India.

3. Junior Resident, Department of Radiodiagnosis, Goa Medical College Bambolim, Goa, India.

4. Professor and Head, Department of Radiodiagnosis, Goa Medical College Bambolim, Goa, India.

NAME, ADDRESS, E-MAIL ID OF THE CORRESPONDING AUTHOR:

Dr. Nilkanth Laxman Pal,

Lecturer, Department of Radiodiagnosis, Goa Medical College, Bambolim, Goa, India. E-mail: nil25.pal@gmail.com

AUTHOR DECLARATION:

- Financial or Other Competing Interests: None

- Was informed consent obtained from the subjects involved in the study? Yes

- For any images presented appropriate consent has been obtained from the subjects. Yes
PLAGIARISM CHECKING METHODS: [Jain H et al.]

- Plagiarism X-checker: Nov 02, 2020

- Manual Googling: Dec 19, 2020

- iThenticate Software: Feb 18, 2021 (8\%)
ETYMOLOGY: Author Origin

Date of Submission: Oct 29, 2020

Date of Peer Review: Dec 07, 2020

Date of Acceptance: Dec 21, 2020

Date of Publishing: Apr 01, 2021 